\title{
Thermodynamic Paradigm for Solution Demixing Inspired by Nuclear Transport in Living Cells
}

\author{
Ching-Hao Wang ${ }^{1, *}$ and Pankaj Mehta ${ }^{1, \dagger}$ \\ ${ }^{1}$ Department of Physics, Boston University, Boston, Massachusetts 02215, USA \\ Michael Elbaum ${ }^{2, \ddagger}$ \\ ${ }^{2}$ Department of Materials and Interfaces, Weizmann Institute of Science, Rehovot, 7600001 Israel
}

(Received 27 July 2016; published 10 April 2017)

\begin{abstract}
Living cells display a remarkable capacity to compartmentalize their functional biochemistry. A particularly fascinating example is the cell nucleus. Exchange of macromolecules between the nucleus and the surrounding cytoplasm does not involve traversing a lipid bilayer membrane. Instead, large protein channels known as nuclear pores cross the nuclear envelope and regulate the passage of other proteins and RNA molecules. Beyond simply gating diffusion, the system of nuclear pores and associated transport receptors is able to generate substantial concentration gradients, at the energetic expense of guanosine triphosphate hydrolysis. In contrast to conventional approaches to demixing such as reverse osmosis and dialysis, the biological system operates continuously, without application of cyclic changes in pressure or solvent exchange. Abstracting the biological paradigm, we examine this transport system as a thermodynamic machine of solution demixing. Building on the construct of free energy transduction and biochemical kinetics, we find conditions for the stable operation and optimization of the concentration gradients as a function of dissipation in the form of entropy production.
\end{abstract}

DOI: 10.1103/PhysRevLett.118.158101

Demixing of solutions is a difficult thermodynamic problem with important practical consequences [1]. Examples include the desalination of seawater, medical dialysis, and chemical purification. In all of these processes, free energy is consumed in order to balance entropy of mixing. Typical engineering approaches to demixing involve an application of hydrostatic pressure (reverse osmosis), a solution exchange (dialysis), or a phase change (crystallization or distillation) [2,3]. In this context living cells adopt a fundamentally different paradigm by establishing and maintaining concentration gradients at steady state under a fixed set of intrinsic thermodynamic parameters. This recalls the similar capacity to operate mechanochemical motors isothermally $[4,5]$.

A prominent example of molecular separation is the eukaryotic cell nucleus, wherein the concentrations of many proteins and RNA differ significantly from those in the cell body (cytoplasm). These gradients are maintained by a transport system that shuttles molecular cargo in and out via large protein channels known as nuclear pores $[6,7]$. This system has been under intensive study in the biological [8-12] and biophysical [13-17] literatures, with particular emphasis on single-molecule interactions at the

Published by the American Physical Society under the terms of the Creative Commons Attribution 4.0 International license. Further distribution of this work must maintain attribution to the author(s) and the published article's title, journal citation, and DOI. pore itself [18-21]. Simple thermodynamic considerations make clear that equilibrium pore-molecule interactions are insufficient to support concentration gradients in solution. Demixing between two compartments cannot occur spontaneously but must be coupled to a free energy source [22]. At the same time, demixing does not require rectified translocation [23]. Concentration gradients may be established in the presence of a balanced, bidirectional exchange [14,24,25].

Nuclear pores represent an unusual transporter in that there is no membrane to cross. Water, ions, and small molecules diffuse freely across the nuclear envelope to equilibrate between the two compartments. Generally, the permeability drops between molecular weights 20 and $40 \mathrm{kDa}[26,27]$. Transport of larger macromolecules relies on a special class of proteins, called transport receptors (i.e., "importin"), that usher their cargoes across the nuclear pores by virtue of specific interactions with the channel components. Recognition between importins and their molecular cargo depends on the presence of particular amino acid sequences known as nuclear localization signals (NLSs) [8,28,29]. The affinity between importin and cargo is regulated by a small guanosine triphosphate (GTP)-binding protein called Ran [30,31]. When associated with GTP (RanGTP), Ran binds strongly to importin in a manner that is competitive with NLS binding. By contrast, Ran associated with guanosine diphosphate (GDP; RanGDP) binds importin very weakly. Ran interconverts between these two forms through GTP hydrolysis and GTP or GDP exchange, facilitated by the GTPase 
(a)
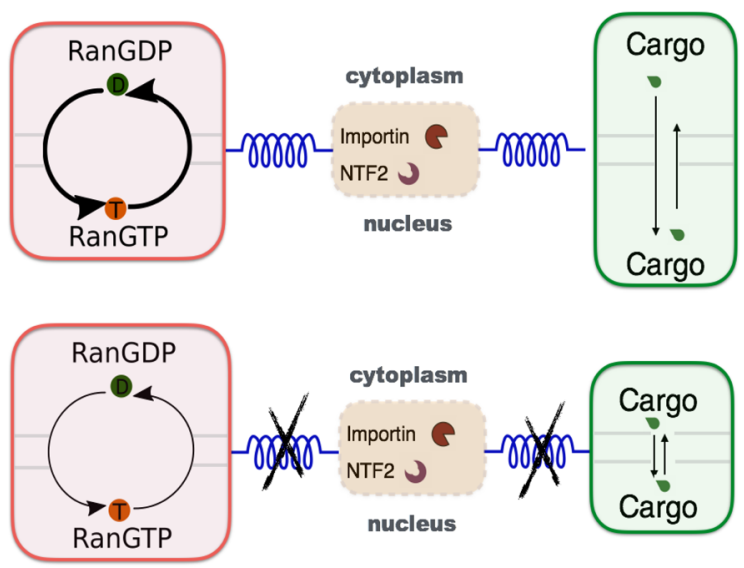

(b)

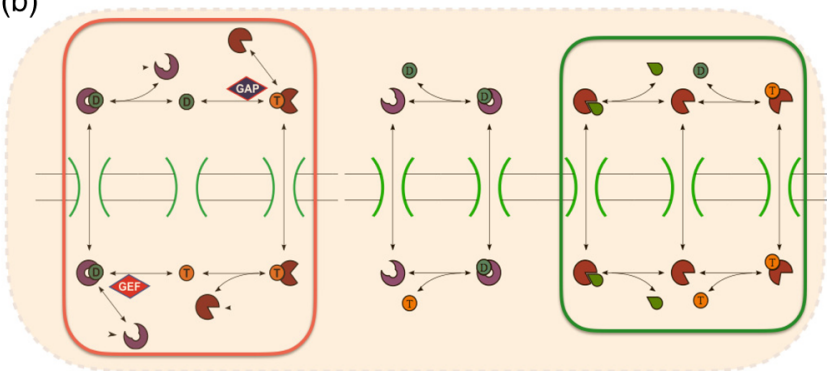

FIG. 1. Demixing of cargo across the nuclear membrane is driven by Ran coupled to a NTF2 and importin system. (a) With such a coupling (upper panel), nuclear cargo accumulation is favored and the Ran GTP or GDP exchange cycle proceeds faster than without coupling (lower panel). The thickness of arrowed curves in the Ran cycle indicates the strength of the reaction flux; the length of the arrowed lines in cargo transport represents the rate at which the underlying processes occur. (b) Details of a molecular demixing machine in the context of nuclear transport. Species labels are as above. Reactions corresponding to Ran cycle and cargo transport are highlighted by red and green boxes, respectively. The orange dashed box includes all reactions coupled with the importin-NTF2 system. See also Fig. S1 in the Supplemental Material [33].

activating protein (RanGAP) and the guanosine exchange factor (RanGEF), respectively [32]. RanGAP is structurally bound to the cytoplasmic face of the nuclear pore and RanGEF is bound to chromatin. Their activities generate a high concentration of RanGTP in the nucleus and RanGDP in the cytoplasm (see Fig. 1).

Demixing is powered by transducing free energy from GTP hydrolysis through the interactions of the transport receptor with Ran. The transport machinery has been formulated in terms of coupled chemical kinetics $[23,34,37]$, but the energetics have not yet been addressed. In particular, we ask: How does the rate of dissipation (energy consumption) relate to the achieved concentration gradient? What is the proper definition of transport efficiency? Is there an optimal working point given the nonequilibrium nature of this cellular machine? To address these questions, it is helpful to reformulate the problem in a thermodynamic language. For consistency with the literature, we retain the biological nomenclature, yet the aim is to understand the natural engineering in a more abstract sense that might ultimately be implemented synthetically.

In the thermodynamic formulation, a central role is played by energy transduction in a "futile cycle" among the components (see Fig. 1). This is roughly analogous to heat flow in a Carnot cycle. The importin receptor binds RanGTP, and a second receptor known as nuclear transport factor 2 (NTF2) binds specifically RanGDP. The forward cycle takes RanGTP out to the cytoplasm with importin and RanGDP back to the nucleus with NTF2. Detailed balance is broken by the distribution of RanGAP and RanGEF as described above, so that the reverse cycle is scarcely populated.

Free energy from the Ran cycle is transduced by importin to bias the steady-state free cargo concentrations in the nuclear and cytoplasmic compartments. Details of the underlying biochemical reactions are shown in Fig. 1(b) and can be modeled on the basis of mass action. The corresponding kinetic parameters can be found in the literature or estimated from simple scaling arguments (see Fig. S1 in the Supplemental Material for details of the kinetic model [33]). Numerical solutions are obtained by solving all of the coupled rate equations using a standard Runge-Kutta method (the code used for simulation is available in the Supplemental Material [33]). We emphasize that the present aim is not so much to model the biological implementation as to explore the generic operation of the thermodynamic machine. The relations between parameters are therefore more important than the specific values.

Energetics enter the model via the charging of Ran with GTP and its subsequent hydrolysis to GDP (reactions 5 and 2 in Fig. S1 [33], respectively). The flux through these two reactions must be equal in steady state. Energy is drawn from the nonequilibrium ratio of free GTP to GDP, $\theta$, which is maintained by cellular metabolism and defines an effective "free energy" $F_{\theta}:=k_{B} T \log (\theta)$. A typical value of $\theta$ is roughly a few tens to a hundred $[32,38]$. Independent of the complex operational details of RanGEF and RanGAP with associated cofactors, we can look at the steady states and relate the reactions to $\theta$. (See the Supplemental Material [33] for details.) On the nuclear side, the complex NTF2-RanGDP exchanges for NTF2 and RanGTP. The dissociation constant $K_{D}$ (forward divided by the reverse flux) can be shown to be proportional to $\theta$. Conversely, on the cytoplasmic side, the corresponding $K_{D}$ is proportional to $1 / \theta$. As a result, any enhancement of flux through the futile cycle in the forward reaction conferred by increasing $\theta$ [i.e., reaction 5 in Fig. S1(c) [33]] is balanced by the contradicting counterpart in preventing a RanGTP release [i.e., reaction 2 in Fig. S1(c) [33]].

A useful measure of cargo demixing is the nuclear localization ratio (NL), defined as the ratio between nuclear and cytoplasmic cargo concentrations: $[C]_{\text {nu }} /[C]_{\text {cyto }}$. This 

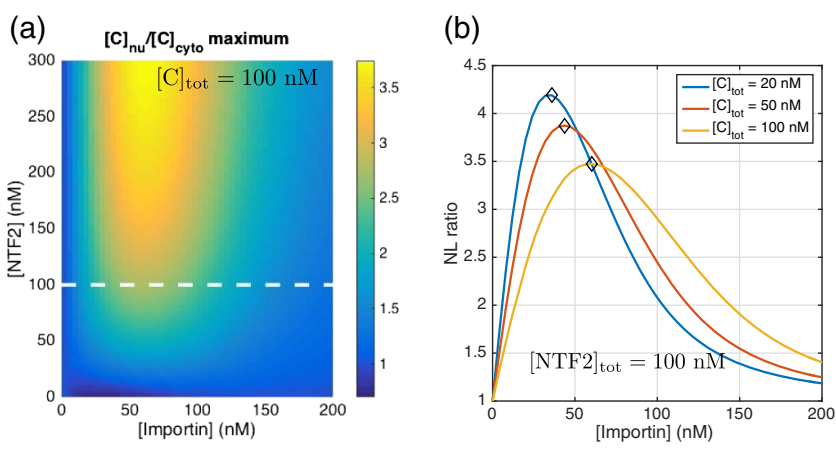

FIG. 2. Phase diagram of nuclear localization. (a) The cargo nuclear localization $\mathrm{NL}:=[C]_{\text {nu }} /[C]_{\text {cyto }}$ (color shadings) is obtained by varying the total importin and total NTF2 concentrations while keeping the overall cargo level fixed at $[C]_{\text {tot }}=100 \mathrm{nM}$. (b) A family of curves shows NL for several cargo concentrations as a function of importin concentration with $[\mathrm{NTF} 2]_{\mathrm{tot}}=100 \mathrm{nM}$. The $1 \mathrm{D}$ curve for $[C]_{\mathrm{tot}}=100 \mathrm{nM}$ is a cut across the plot of (a). Locations of the NL maximum are marked by diamonds [see Fig. 4(c) as well]. Kinetic rate constants used are given in the Supplemental Material [33]. The total Ran concentration is $[\operatorname{Ran}]_{\mathrm{tot}}=75 \mathrm{nM}$.

ratio defines a chemical potential, $\Delta \mu=-k_{B} T \log [C]_{\mathrm{nu}} /$ $[C]_{\text {cyto }}$, that measures the excursion from equilibrium. Figure 2(a) shows NL as a function of importin and NTF2 concentrations. The most striking feature is that $\mathrm{NL}$ is maximum for intermediate levels of importin. The importin concentration at which NL is maximized, [ $\mathrm{Im}^{*}$, grows with the total cargo load, $[C]_{\text {tot }}$ [see Fig. 2(b)]. Furthermore, $\left[\mathrm{Im}^{*}\right]$ is largely independent of NTF2 concentration for the different cargo concentrations considered (see Fig. S5 [33]). This suggests an inherent optimization.

At first sight it is surprising that augmenting the importin concentration, which increases the number of molecules that can transport cargo to nucleus, may decrease the localization ratio. The optimal dependence of NL on importin reflects the dual role importin plays as the inbound carrier of cargo protein as well as the outbound carrier of RanGTP. Powering the futile cycle requires that importin bind RanGTP, whereas cargo transport requires importin to bind cargo. This establishes a binding competition in the nucleus that is a characteristic feature of protein import [Fig. 3(a)]. In spite of the higher affinity of RanGTP for importin, the cycle analysis shows that importin in the nucleus binds cargo more rapidly. As seen in Figs. 3(b) and 3(c), NL is maximized close to the point at which the difference between the reaction fluxes of the importincargo formation $\left(\Phi_{7}^{-}:=\tilde{\Phi}_{7}^{-}[\operatorname{Im}]_{\mathrm{nu}}=\left(k_{7}^{-}[C]_{\mathrm{nu}}\right)[\operatorname{Im}]_{\mathrm{nu}}\right)$ and the importin-RanGTP formation $\left(\Phi_{4}^{+}:=\tilde{\Phi}_{4}^{+}[\mathrm{Im}]_{\mathrm{nu}}=\right.$ $\left.\left(k_{4}^{+}[\operatorname{RanGTP}]_{\mathrm{nu}}\right)[\mathrm{Im}]_{\mathrm{nu}}\right)$ is maximal. Intuitively, this is the realm where importin can bind cargo effectively while maintaining its coupling to the reaction cycle that transduces energy for cargo transport.

To understand the thermodynamics of nuclear transport, we formulate the transport system as a nonequilibrium (a)

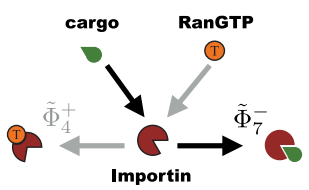

(b)

(c)
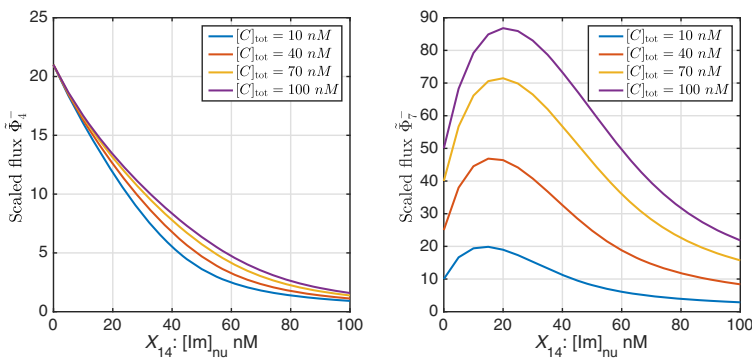

FIG. 3. Competition between RanGTP and cargo to bind importin. (a) Schematic of the two competing reactions. (b) Reaction flux for the importin-RanGTP formation $\tilde{\Phi}_{4}^{+} \sim$ $k_{4}^{+}[$RanGTP $]$and (c) flux for importin-cargo formation $\tilde{\Phi}_{7}^{-} \sim k_{7}^{-}[C]_{\text {nu }}$. Fluxes are scaled by $[\operatorname{Im}]_{\text {nu }}$ (see the text). Parameters are as in Fig. 2.

Markov process. Since a nonequilibrium steady state (NESS) necessarily breaks detailed balance in the underlying Markov process, the system has a nonzero entropy production $[22,39,40]$. This is the energy per unit time required to maintain the NESS, with units of power. Following the Schnakenberg description, the entropy production (EP) for a NESS is given by [36]

$$
\mathrm{EP}=k_{B} T \sum_{i, j} P_{i}^{\mathrm{SS}} W(i, j) \log \frac{W(i, j)}{W(j, i)}
$$

where $P_{i}^{\mathrm{SS}}$ is the steady-state probability distribution of state $i$, while $W(i, j)$ denotes the transition probability from state $i$ to state $j$. Concretely, $P_{i}^{S S}$ is the fraction of reactants that participate in the transition reaction starting from state $i$, while $W(i, j)$ can be calculated from the relevant reaction fluxes. Note that the sum in Eq. (1) is taken over all links of the reaction network. This is equivalent to summing over the links pertaining to the Ran futile cycle. (See the Supplemental Material [33] for details).

This entropy production provides a direct measure of the power input to the underlying biochemical circuit. Figure 4(a) shows the EP for various importin and NTF2 concentrations. Figure 4(b) adds various cargo concentrations for a fixed level of [NTF2]. In each case, as the importin concentration increases, EP first drops to a minimum and then peaks before slowly decaying. Note that the minimal dissipation (entropy production) tracks closely with the value at which the NL ratio peaks [see Fig. 4(c)]. These conditions define an optimal efficiency of the demixing machine. With a further increased importin concentration, the futile cycle decouples from the cargo translocation and EP increases. At a still higher 
(a)

(c)
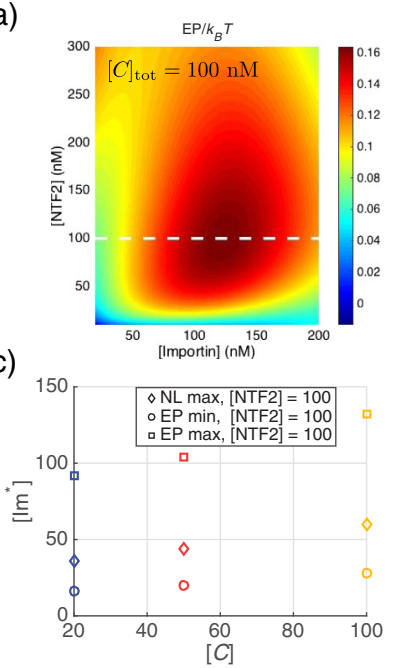

(b)

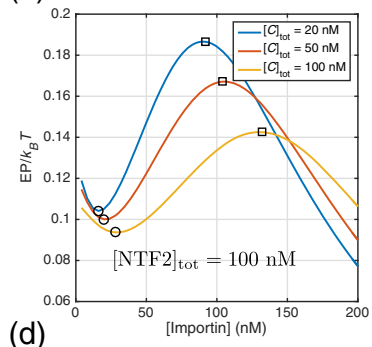

(d)

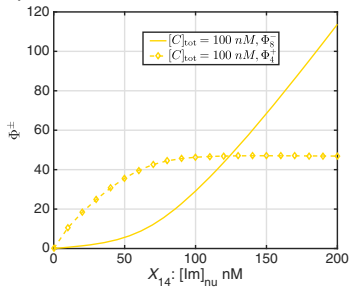

FIG. 4. Phase diagram of entropy production. (a) Entropy production is plotted as a 2D function of NTF2 and importin at a fixed cargo concentration $[C]_{\text {tot }}=100 \mathrm{nM}$. Compare with Fig. 2(a). (Axes extend to 5 instead of $0 \mathrm{nM}$ to avoid numerical divergence.) (b) A family of curves shows the entropy production for several cargo concentrations as a function of importin concentration; NTF2 concentration is fixed at $[\mathrm{NTF} 2]_{\text {tot }}=$ $100 \mathrm{nM}$. The $1 \mathrm{D}$ curve for $[C]_{\text {tot }}=100 \mathrm{nM}$ is a cut across the plot of (a). Compare with Fig. 2(b). Peaks and troughs are marked by squares and circles, respectively. (c) A square (circle) indicates the location of the entropy production maximum (minimum), while a diamond marks the nuclear localization maximum. Colors match the curves in (b). The importin concentration at which EP is minimal is close to but always less than [Im*], where $\mathrm{NL}$ is maximal. Thus, one strategy for maximizing the efficiency of demixing is to have the futile cycle operate in a regime where its entropy production is minimized. (d) EP decreases at very high importin concentrations. This reflects a loop around the energetic reaction $\Phi_{4}$ via the reversible reaction $\Phi_{8}$. Here, $[\mathrm{NTF} 2]_{\text {tot }}=$ $100 \mathrm{nM}$, as in (b). In all panels, $[\operatorname{Ran}]_{\text {tot }}=75 \mathrm{nM}$. The kinetic constants are as in Figs. 2 and 3 (see Sec. II of the Supplemental Material [33]).

concentration, EP peaks and then decreases. This can be understood qualitatively as a short circuit via reaction 8 , where importin moves between compartments carrying neither cargo nor RanGTP. As seen in Fig. 4(a), for such high importin levels, the corresponding flux $\Phi_{8}$ exceeds that of the RanGTP loading to importin, $\Phi_{4}$.

To the best of our knowledge, the optimal steady state has not been observed experimentally. The kinetic rate of the nuclear protein uptake was found to be reduced by the microinjection of an importin receptor to live cells; rate equation simulations done in parallel also pointed to the dual role of importin [Fig. 3(a)] [37]. Steady states were not analyzed, however. Other possible experimental tests include titration of importin protein to nuclei reconstituted in vitro in Xenopus egg extract and optical activation of importin receptors, similar to the induction of nuclear transport by NLS activation [41]. An important point in

comparison with the literature is that we have considered a single, collective "cargo"for transport. In reality, multiple cargoes compete for binding to relatively few but promiscuous transport receptors. This competition leads to a partitioning according to equilibrium binding affinities and may lead to vastly different kinetics. However the steady-state NL ratio (in solution) is independent of the affinity, reflecting thermodynamic control and equilibration of the chemical potentials [24,25,34]. Consistent with this paradigm, in which a net accumulation occurs together with a balanced bidirectional flux, the simulations show that the nuclear and cytoplasmic concentrations of the importincargo complex ( $X_{4}$ and $X_{11}$, respectively) equilibrate in steady state. It is also interesting to note that RanGTP loading onto importin (reaction 4) was identified in the earlier analysis as the primary rate-limiting step in accumulation kinetics [34].

In summary, we have analyzed the biological paradigm for nuclear transport from a thermodynamic point of view. Building upon the prior understanding that protein cargo demixing is facilitated by hydrolysis of GTP, we draw the connection between the consumption of chemical energy and maintenance of the cargo concentration gradient at nonequilibrium steady states. We show that the efficacy of nuclear localization ratio peaks at an intermediate importin level, which is not far from the power consumption (entropy production) minimum. It is likely that the cell maintains an importin concentration at an advantageous level with respect to these operating points that is defined by the thermodynamic analysis. Interestingly, the system as configured is robust to the quality of the chemical energy source, in the sense that the NL ratio is almost independent of the GTP-to-GDP ratio $\theta$ when $\theta \gtrsim 20$ (see Fig. S4 of the Supplemental Material [33]). A thermodynamic definition of the system efficiency remains elusive, however. Whereas conventional efficiency of an engine is a dimensionless ratio of mechanical to thermal power, in the NESS a constant free energetic gradient (chemical potential, in the present case) is maintained by a constant power input. The ratio has units of time. This could be renormalized sensibly by a characteristic remixing time, e.g., the permeability of the nuclear pores to the cargo-importin complex. There is no guarantee of a bound at unity, however, so the definition remains ad hoc, a useful figure of merit. It is also interesting to contrast the competitive interactions between the receptor and RanGTP in nuclear protein accumulation (import) with the cooperative interactions in nuclear protein depletion (export). While these are often considered simple inverse processes, they differ in this essential aspect [42].

This work is part of a larger body of literature that seeks to examine basic biophysical processes from a thermodynamic perspective. It is now clear that thermodynamics fundamentally constrains the ability of cells to perform various tasks, ranging from external signal detection [43-45] to adaptation [46] to making fidelity decisions [40], generating oscillatory 
behavior [47], and, of course, generating forces and dynamic structures [48-50]. In all of these examples, it is possible to map these tasks to Markov processes and compute the corresponding entropy production rate. This suggests that there may be general theorems about thermal efficiency in cells that are independent of the particular task under consideration [39,51-53]. It will be interesting to explore whether this is actually the case and to see if these principles can be applied to synthetic biology and, ultimately, biomimetic engineering [51].

P. M. and C.-H.W. were supported by a Simons Investigator in the Mathematical Modeling of Living Systems grant, a Sloan Fellowship, and NIH Grant No. 1R35GM119461 (all directed to P. M.). M. E. acknowledges a grant from the Israel Science Foundation (Grant No. 1369/10), the Gerhardt M. J. Schmidt Minerva Research Foundation, and the historical generosity of the Harold Perlman family. Simulations were carried out on the Shared Computing Cluster (SCC) at BU.

*chinghao@bu.edu

pankajm@bu.edu

*michael.elbaum@weizmann.ac.il

[1] M. Dijkstra and D. Frenkel, Evidence for Entropy-Driven Demixing in Hard-Core Fluids, Phys. Rev. Lett. 72, 298 (1994).

[2] K. H. Mistry, R. K. McGovern, G. P. Thiel, E. K. Summers, S. M. Zubair, and J. H. Lienhard, Entropy generation analysis of desalination technologies, Entropy 13, 1829 (2011).

[3] P. D. Glynn and E. J. Reardon, Solid-solution aqueoussolution equilibria; thermodynamic theory and representation, Am. J. Sci. 290, 164 (1990).

[4] A. Parmeggiani, F. Jülicher, A. Ajdari, and J. Prost, Energy transduction of isothermal ratchets: Generic aspects and specific examples close to and far from equilibrium, Phys. Rev. E 60, 2127 (1999).

[5] J. Parrondo, B. de Cisneros, and R. Brito, Thermodynamics of isothermal Brownian motors, in Stochastic Processes in Physics, Chemistry, and Biology, edited by J. A. Freund and T. Pöschel, Lecture Notes in Physics Vol. 38 (Springer, New York, 2000).

[6] G. G. Maul and L. Deaven, Quantitative determination of nuclear pore complexes in cycling cells with differing DNA content, J. Cell Biol. 73, 748 (1977).

[7] B. Talcott and M. S. Moore, Getting across the nuclear pore complex, Trends Cell Biol. 9, 312 (1999).

[8] D. Görlich and U. Kutay, Transport between the cell nucleus and the cytoplasm, Annu. Rev. Cell Dev. Biol. 15, 607 (1999).

[9] M. Stewart, Molecular mechanism of the nuclear protein import cycle, Nat. Rev. Mol. Cell Biol. 8, 195 (2007).

[10] M. A. D'Angelo and M. W. Hetzer, Structure, dynamics and function of nuclear pore complexes, Trends Cell Biol. 18, 456 (2008).
[11] S. R. Wente and M. P. Rout, The nuclear pore complex and nuclear transport, Cold Spring Harbor Perspect. Biol. 2, a000562 (2010).

[12] M. Kimura and N. Imamoto, Biological significance of the importin- $\beta$ family-dependent nucleocytoplasmic transport pathways, Traffic 15, 727 (2014).

[13] R. Peters, Optical single transporter recording: transport kinetics in microarrays of membrane patches, Annu. Rev. Biophys. Biomol. Struct. 32, 47 (2003).

[14] R. B. Kopito and M. Elbaum, Reversibility in nucleocytoplasmic transport, Proc. Natl. Acad. Sci. U.S.A. 104, 12743 (2007).

[15] R. S. Wagner, L. E. Kapinos, N. J. Marshall, M. Stewart, and R. Y. Lim, Promiscuous binding of karyopherin $\beta 1$ modulates FG nucleoporin barrier function and expedites NTF2 transport kinetics, Biophys. J. 108, 918 (2015).

[16] R. Zahn, D. Osmanović, S. Ehret, C. A. Callis, S. Frey, M. Stewart, C. You, D. Görlich, B. W. Hoogenboom, and R. P. Richter, A physical model describing the interaction of nuclear transport receptors with FG nucleoporin domain assemblies, eLife 5, e14119 (2016).

[17] A. Vovk, C. Gu, M. G. Opferman, L. E. Kapinos, R. Y. Lim, R. D. Coalson, D. Jasnow, and A. Zilman, Simple biophysics underpins collective conformations of the intrinsically disordered proteins of the Nuclear Pore Complex, eLife 5, e10785 (2016).

[18] O. Keminer and R. Peters, Permeability of single nuclear pores, Biophys. J. 77, 217 (1999).

[19] W. Yang, J. Gelles, and S. M. Musser, Imaging of singlemolecule translocation through nuclear pore complexes, Proc. Natl. Acad. Sci. U.S.A. 101, 12887 (2004).

[20] U. Kubitscheck, D. Grünwald, A. Hoekstra, D. Rohleder, T. Kues, J. P. Siebrasse, and R. Peters, Nuclear transport of single molecules, J. Cell Biol. 168, 233 (2005).

[21] D. Grünwald, R. H. Singer, and M. Rout, Nuclear export dynamics of RNA-protein complexes, Nature (London) 475, 333 (2011).

[22] T. L. Hill, Free Energy Transduction and Biochemical Cycle Kinetics (Springer Science+Business Media, Berlin Heidelberg, 2000), Vol. 557.

[23] D. Görlich, M. J. Seewald, and K. Ribbeck, Characterization of Ran-driven cargo transport and the RanGTPase system by kinetic measurements and computer simulation, EMBO J. 22, 1088 (2003).

[24] R. B. Kopito and M. Elbaum, Nucleocytoplasmic transport: a thermodynamic mechanism, HFSP J. 3, 130 (2009).

[25] O. Lolodi, H. Yamazaki, S. Otsuka, M. Kumeta, and S. H. Yoshimura, Dissecting in vivo steady-state dynamics of karyopherin-dependent nuclear transport, Mol. Biol. Cell 27, 167 (2016).

[26] R. Peters, Nucleo-cytoplasmic flux and intracellular mobility in single hepatocytes measured by fluorescence microphotolysis, EMBO J. 3, 1831 (1984).

[27] A. Samudram, B. M. Mangalassery, M. Kowshik, N. Patincharath, and G. K. Varier, Passive permeability and effective pore size of HeLa cell nuclear membranes, Cell. Biol. Int. 40, 991 (2016).

[28] M. Grote, U. Kubitscheck, R. Reichelt, and R. Peters, Mapping of nucleoporins to the center of the nuclear pore 
complex by post-embedding immunogold electron microscopy, J. Cell Sci. 108, 2963 (1995).

[29] M. Rexach and G. Blobel, Protein import into nuclei: association and dissociation reactions involving transport substrate, transport factors, and nucleoporins, Cell 83, 683 (1995).

[30] A. E. Smith, B. M. Slepchenko, J. C. Schaff, L. M. Loew, and I. G. Macara, Systems analysis of Ran transport, Science 295, 488 (2002).

[31] L. J. Terry, E. B. Shows, and S. R. Wente, Crossing the nuclear envelope: hierarchical regulation of nucleocytoplasmic transport, Science 318, 1412 (2007).

[32] J. L. Bos, H. Rehmann, and A. Wittinghofer, GEFs and GAPs: critical elements in the control of small G proteins, Cell 129, 865 (2007).

[33] See Supplemental Material at http://link.aps.org/ supplemental/10.1103/PhysRevLett.118.158101, which includes Refs. [30,34-36], for details of the model and additional figures.

[34] S. Kim and M. Elbaum, A simple kinetic model with explicit predictions for nuclear transport, Biophys. J. 105, 565 (2013).

[35] M. T. Harreman, P. E. Cohen, M. R. Hodel, G. J. Truscott, A. H. Corbett, and A. E. Hodel, Characterization of the auto-inhibitory sequence within the N-terminal Domain of importin $\alpha$, J. Biol. Chem. 278, 21361 (2003).

[36] J. L. Lebowitz and H. Spohn, A Gallavotti-Cohen-type symmetry in the large deviation functional for stochastic dynamics, J. Stat. Phys. 95, 333 (1999).

[37] G. Riddick and I. G. Macara, A systems analysis of importin- $\alpha-\beta$ mediated nuclear protein import, J. Cell Biol. 168, 1027 (2005).

[38] R. H. Garrett and C. M. Grisham, Biochemistry (BrooksCole, Belmont, MA, 2008).

[39] P. Mehta and D. J. Schwab, Energetic costs of cellular computation, Proc. Natl. Acad. Sci. U.S.A. 109, 17978 (2012).

[40] A. H. Lang, C. K. Fisher, T. Mora, and P. Mehta, Thermodynamics of Statistical Inference by Cells, Phys. Rev. Lett. 113, 148103 (2014).
[41] D. Niopek, D. Benzinger, J. Roensch, T. Draebing, P. Wehler, R. Eils, and B. Di Ventura, Engineering light-inducible nuclear localization signals for precise spatiotemporal control of protein dynamics in living cells, Nat. Commun. 5, 4404 (2014).

[42] S. Kim and M. Elbaum, Enzymatically driven transport: a kinetic theory for nuclear export, Biophys. J. 105, 1997 (2013).

[43] H. C. Berg and E. M. Purcell, Physics of chemoreception, Biophys. J. 20, 193 (1977).

[44] R. G. Endres and N. S. Wingreen, Maximum Likelihood and the Single Receptor, Phys. Rev. Lett. 103, 158101 (2009).

[45] T. Mora and N. S. Wingreen, Limits of Sensing Temporal Concentration Changes by Single Cells, Phys. Rev. Lett. 104, 248101 (2010).

[46] P. Sartori, L. Granger, C. F. Lee, and J. M. Horowitz, Thermodynamic costs of information processing in sensory adaptation, PLoS Comput. Biol. 10, e1003974 (2014).

[47] M. B. Elowitz and S. Leibler, A synthetic oscillatory network of transcriptional regulators, Nature (London) 403, 335 (2000).

[48] F. Nédélec, T. Surrey, A. C. Maggs, and S. Leibler, Selforganization of microtubules and motors, Nature (London) 389, 305 (1997).

[49] T. Surrey, F. Nédélec, S. Leibler, and E. Karsenti, Physical properties determining self-organization of motors and microtubules, Science 292, 1167 (2001).

[50] E. Karsenti, Self-organization in cell biology: a brief history, Nat. Rev. Mol. Cell Biol. 9, 255 (2008).

[51] P. Mehta, A. H. Lang, and D. J. Schwab, Landauer in the age of synthetic biology: energy consumption and information processing in biochemical networks, J. Stat. Phys. 162, 1153 (2016).

[52] A. C. Barato and U. Seifert, Thermodynamic Uncertainty Relation for Biomolecular Processes, Phys. Rev. Lett. 114, 158101 (2015).

[53] T. R. Gingrich, J. M. Horowitz, N. Perunov, and J. L. England, Dissipation Bounds All Steady-State Current Fluctuations, Phys. Rev. Lett. 116, 120601 (2016). 